\title{
SHP2 Inhibitor JAB-3068
}

National Cancer Institute

\section{Source}

National Cancer Institute. SHP2 Inhibitor IAB-3068. NCI Thesaurus. Code C160207.

An orally bioavailable inhibitor of protein tyrosine phosphatase (PTP) non-receptor type

11 (SHP2; Src homology region 2 domain phosphatase; PTPN11), with potential

antineoplastic activity. Upon oral administration, SHP2 inhibitor JAB-3068 targets, binds to and inhibits the activity of SHP2. This prevents SHP2-mediated signaling, inhibits MAPK signaling and prevents growth of SHP2-expressing tumor cells. SHP2, an oncoprotein overexpressed in a variety of cancer cell types, regulates cell survival, differentiation and proliferation through activation of the Ras-Raf-MEK-ERK signaling pathway. The RasMAPK pathway is often hyperactivated in cancer cells due to specific mutations and rearrangements and are dependent on SHP2 for their oncogenic signaling. SHP2 also regulates programmed cell death 1 (PD-1)-mediated signal transduction and is involved in immune checkpoint modulation. 